\title{
Long term effects of mild severity COVID-19 on right ventricular functions
}

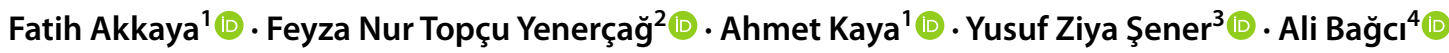

Received: 5 June 2021 / Accepted: 2 July 2021 / Published online: 12 July 2021

(c) The Author(s), under exclusive licence to Springer Nature B.V. 2021

\begin{abstract}
Coronavirus disease 2019 (COVID-19) is a newly recognized infectious disease that has spread rapidly. COVID-19 has been associated with a number of cardiovascular involvements, including ventricular functions. The aim of our study was to evaluate the right ventricular functions of mild severity COVID-19 patients 3 months after, and compare them to the right ventricular functions of healthy volunteers. For this single-center study, data from 105 patients who were treated for mild severity COVID-19 between September 15, 2020 and December 31, 2020 were collected. 105 age and sex matched healthy subjects were included in the study. Right ventricular (RV) functions were evaluated using conventional two-dimensional (2D) echocardiography and 2D speckle-tracking echocardiography (STE) for all patients. 2D-E parameters indicating RV functions were compared between the two groups. RV diamaters, systolic pulmonary artery pressure (sPAP) and RV myocardial performance index (RV MPI) were significantly higher in the COVID-19 patients compared to control group $(\mathrm{p}<0.05)$. Tricuspid annular plane systolic motion (TAPSE), right ventricular fractional area change (RVFAC) and RV S' were significantly lower in the COVID-19 group compared to control group $(\mathrm{p}<0.05)$. RV global longitudinal strain (RVGLS) $(-19.6 \pm 5.2$ vs. $-15.1 \pm 3.4, \mathrm{p}<0.001)$ and RV free wall longitudinal strain RV-FWLS $(-19.6 \pm 5.2$ vs. $-17.2 \pm 4.4$, $\mathrm{p}<0.001)$ values were significantly lower in the COVID-19 group than the control group. There was a significant negative correlation between RV-FWLS, RV-GLS and C-reactive protein (CRP), neutrophil to lymphocyte ratio (NLR), d-dimer, ferritin, platelet to lymphocyte ratio (PLR) in patients with mild severity COVID-19. This results suggested that RV-GLS and RV-FWLS decreased in the long term (third month) follow-up of patients treated for mild severity COVID-19 disease. Subclinical RV dysfunction may be observed in patients after mild severity COVID-19.
\end{abstract}

Keywords Coronavirus disease $2019 \cdot$ Right ventricular function $\cdot$ Speckle tracking echocardiography

Yusuf Ziya Şener

yzsener@yahoo.com.tr

Fatih Akkaya

drfatihakkaya@gmail.com

Feyza Nur Topçu Yenerçağ

fntopcu@hotmail.com

Ahmet Kaya

doktorahmetkaya@yahoo.com

Ali Bağcı

dr.alibagci@ hotmail.com

1 Department of Cardiology, Ordu University, Ordu, Turkey

2 Provincial Health Directorate, Ordu, Turkey

3 Department of Cardiology, Beypazarı State Hospital, Ankara, Turkey

4 Department of Cardiology, Suleyman Demirel University, Isparta, Turkey

\section{Introduction}

Coronavirus disease 2019 (COVID-19) was first appeared at the end of 2019 in the Wuhan city which is the capital city of Hubei region of China and led to pandemic. COVID-19 which develops due to infection with severe acute respiratory syndrome- Coronavirus -2 (SARS-CoV-2) resulted in high rates of morbidity and mortality worldwide [1]. Although the main target of the disease is respiratory tract; COVID-19 may affect all of the organ systems such as cardiovascular system. Microthrombogenesis due to hypercoagulapathy, increased systemic inflammatory response, hypoxia, and hypotension are thought to play major role in the pathophysiology of cardiac involvement following SARS-CoV-2 infection [2].

Severe systemic inflammatory response and hypercoagulopathy may develop in several contagious infectious 
diesases including COVID-19 and this situation may result in increased levels of C-reactive protein, ferritin, D-dimer, neutrophil to lymphocyte ratio [3, 4]. Complex systemic inflammatory response may last long and affect ventricular functions. Several studies including COVID-19 patients in which right ventricular functions were evaluated by echocardiography in the early stages of the disease demonstrated that COVID-19 affects right ventricular functions [5-7]. However, there is no data about the long term effects of mild severity COVID-19 disease on right ventricular functions in the literature.

In this study, we aimed to evaluate long term effects of mild severity COVID-19 disease on right ventricular functions.

\section{Material and methods}

\section{Study population and procedure}

This cross-sectional, single-centre study was performed in University of Ordu Training and Research Hospital, which was determined to treat COVID-19 patients by Turkish Republic Ministry of Health, between November 2020 and December 2020. Patients with mild severity COVID19 disease who were older than 18 years old and treated with only favipravir were included in the study. The mild severity COVID-19 patients; was defined as patients who were followed outpatient, non-hospitalized, and who had constituional symptoms including fever, muscle and/or joint pain, cough, sore throat, without respiratory distress [respiratory rate $<24 / \mathrm{min}$, peripheral capillary oxygen saturation $\left(\mathrm{SpO}_{2}\right)>93 \%$ at room air] according to the World Health Organization and the Republic of Turkey Ministry of Health COVID-19 Treatment Guidelines [8, 9]. Patients with a history of coronary artery disease, chronic inflammatory disease, hypertension, severe chronic renal and liver failure, right or left ventricular failure, atrial fibrillation, complete or incomplete branch block, moderate-severe valvular pathology, pacemaker, diabetes mellitus, anemia, chronic renal failure, thyroid dysfunction, pulmonary hypertension, pulmonary embolism, cancer, chronic lung disease, body mass index $(\mathrm{BMI})>30 \mathrm{~kg} / \mathrm{m}^{2}$ and patients diagnosed with acute coronary syndrome and myocarditis were excluded. In addition, patients who were given steroids and/or heparin along with favipiravir due to COVID-19 and those who had any other active infection disease and used antibiotics were excluded. After the exclusion criterias were applied, the study continued with 105 non-hospitalized adult COVID19 patients. A total of 105 sex- and age-matched healthy subjects were randomly selected as the control group. Demographic data, baseline characteristics, medical history, drugs and medications used for COVID-19, smoking habits and laboratory values were obtained using the hospital's medical database. Laboratory parameters including; complete blood count (CBC), C-reactive protein (CRP), D-dimer, ferritin and other biochemical parameters were measured at the first admission to the hospital before COVID-19 treatment started. The entire study population were evaluated via two-dimensional echocardiography and speckle tracking echocardiographic imaging. The patients' echocardiographic examinations were performed 3 months after COVID19 diagnosis. The research procedures were revised and approved by the local hospital's ethics committee and Ministry of Health Scientific Research Platform according to the ethical considerations stipulated in the Helsinki Declaration.

\section{Diagnosis of COVID-19}

According to the World Health Organization [8] and the Republic of Turkey Ministry of Health COVID-19 Treatment Guidelines [9], patients who were matching the definition of probable SARS-CoV-2 infection underwent testing with molecular methods to scan for viruses. Throat and nasopharynx swab samples were collected from all patients in our study to extract SARS-CoV-2 RNA. Real-time reverse transcription polymerase chain reaction assay (RTPCR) molecular method was applied for RNA analysis of SARS-CoV-2 virus. RT-PCR assay was performed using the SARS-CoV-2 (2019-nCoV) qPCR Detection Kit (Bioeksen R\&D Technologies Co Ltd, Istanbul, Turkey). Cases with SARS-CoV-2 RNA in RT-PCR method were accepted as COVID-19.

\section{Two-dimensional echocardiography (2D-E)}

Bedside transthoracic echocardiographic examinations were performed in all patients using the EPIQ 7C ultrasound system (Philips Medical Systems, Andover, Massachusetts). Two-dimensional and Doppler echocardiography were performed on the basis of the guidelines of the American Society of Echocardiography [10]. All these echocardiographic examinations were performed by experienced two operators. Echocardiographic examinations were performed in left lateral decubitus position after resting for at least $15 \mathrm{~min}$. All measurements were taken in three consecutive cycles, and average values were calculated. Parasternal long and short axis views and apical views were used as standard imaging windows. Left ventricular (LV) end-diastolic/end-systolic diameters (LVEDd, LVESd) were measured using M-mode with the parasternal long axis view and thereafter from apical fourchamber and biplane window views were used to measure left ventricular end-diastolic and end-systolic volume (LVEDV, LVESV). Left ventricular ejection fraction (LVEF) was calculated from the apical window using 
the modified Simpson method. Left atrium diameter was determined from M-mode echocardiographic images using a leading-edge-to-leading-edge method, measuring the maximal distance between the posterior aortic root wall and the posterior left atrial wall at end-systole.Peak velocities of the early diastolic (E) and late diastolic (A) waves were measured at the point of mitral leaflet coaptation in the apical 4-chamber views for the evaluation of diastolic functions. The peak velocities of early diastolic waves (septal $\mathrm{e}^{\prime}$ and lateral $\mathrm{e}^{\prime}$ ) were measured by PW tissue doppler imaging (TDI) from the lateral and septal mitral annulus. E/e'(lateral) ratio was calculated.

Right ventricular (RV) diameters were measured at RV mid-region and basal region from apical four-chamber view. Percentage right ventricular fractional area change (RV-FAC) was calculated by dividing the difference in $\mathrm{RV}$ area between the end diastolic and end-systolic phases by end-diastolic RV area. Tricuspid annular plane systolic excursion (TAPSE) is defined as the distance traveled between end-diastole and end-systole at the lateral corner of the tricuspid annulus. Systolic pulmonary artery pressure (sPAP) was calculated as the sum of right atrial pressure value obtained by Bernoulli's equation from tricuspid valve pressure gradient and caval respiratory index. Calculation of the RV myocardial performance index (RV MPI) was assessed by PW tissue doppler imaging (TDI). To measure RV S', RV-focused view is used with tissue doppler region of interest placed at the lateral corner of the tricuspid annulus acquired at high frame rate. The velocity $S^{\prime}$ is read as the highest systolic velocity. By PW TDI the encompasses isovolumetric contraction time, ejection time (ET), and isovolumetric relaxation time. TDI RV-MPI were measured using the Tei index. Intraobserver and interobserver variations for echocardiographic measurements were less than $4 \%$.

\section{Assessment of right ventricule speckle tracking echocardiography (RV-STE)}

While performing RV-2D strain imaging; the patient's heart rhythm was monitored with echocardiography, 2D video data were recorded from the modified apical 4-chamber (A4C) view, and RV-focused images including at least three cardiac cycles with regular ECG signals were obtained in the tissue velocity imaging mode. The off-line analysis of recorded image sequences and signals was performed using the commercially software (QLAB-CMQ, Philips Healthcare, Andover, Massachusetts) on a computer workstation. After defining three reference landmarks (RV apex, medial and lateral tricuspid annulus), the software automatically traced the endocardial and epicardial borders in the modified A4C view. Tracking points were adjusted manually if necessary, and 2D longitudinal strain and strain rate curves were obtained for each myocardial segment. Peak negative longitudinal systolic strain variables were derived from these curves. RV global longitudinal strain (RV-GLS) and RV free-wall longitudinal strain (RV-FWLS) values were measured according to the current guidelines [11]. 2D speckle-tracking strain images of right ventricle is presented in Fig. 1.

\section{Statistical analysis}

All data were analysed using SPSS (Statistical Package for Social Sciences) software version 22.0. Quantitative variables with a normal distribution were specified as means \pm standard deviation. Categorical variables were shown as number and percentage values. In order to test normality of distribution Kolmogorov-Smirnov test was used. For comparison of quantitative data, student-t test (normally distributed data) and Mann Whitney-U test (nonnormally distributed data) were used. Categorical variables
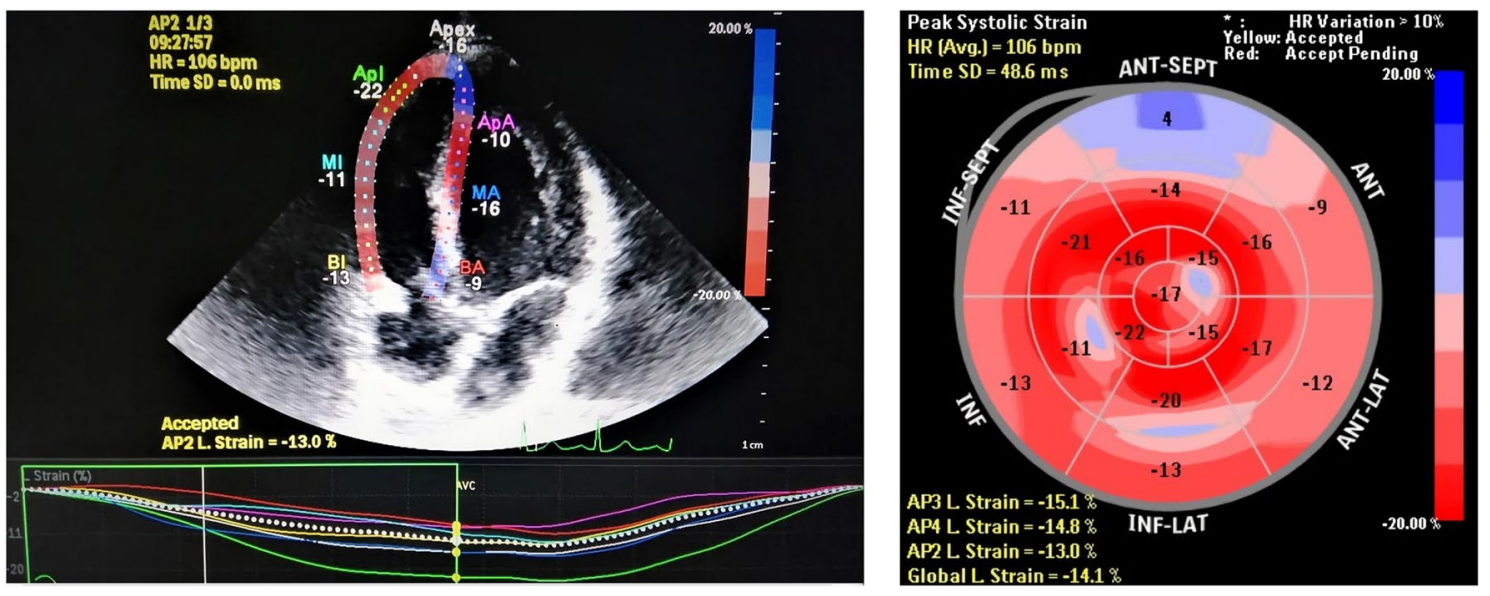

Fig. 1 An example of right ventricular strain speckle tracking apical four chamber view of COVID-19 patient 
were compared with the Chi-square test. Spearman's correlation coefficient tests were used to assess the strength of the relationship between RV strain parameters and CRP, NLR, PLR, LMR, ferritin, D-dimer values. The results were evaluated within a $95 \%$ confidence interval. A p value $<0.05$ was considered to indicate statistical significance.

\section{Results}

Demographic, clinical and laboratory characteristics of the study groups are shown in Table 1. The mean age of the participants was $43.5 \pm 12.5$ years and $60.9 \%$ of the cases were male. There were no significant differences between the two groups in terms of baseline demographic characteristics and medical history $(\mathrm{p}>0.05)$. In their laboratory findings white blood cell $\left(8 \pm 3.210^{3} \mu \mathrm{L}\right.$ vs. $\left.6.4 \pm 1.610^{3} \mu \mathrm{L} ; \mathrm{p}<0.001\right)$, neutrophil $\left(5.5 \pm 2.210^{3} \mu \mathrm{L}\right.$; vs. $\left.4.1 \pm 2.210^{3} \mu \mathrm{L} ; \mathrm{p}<0.001\right)$ and Neutrophil to lymphocyte ratio (NLR) $(6.1 \pm 2.7$ vs.

Table 1 Baseline characteristics and laboratory findings of the study population

\begin{tabular}{|c|c|c|c|}
\hline Parameters & $\begin{array}{l}\text { Control } \\
(n=105)\end{array}$ & $\begin{array}{l}\text { COVID-19 } \\
(\mathrm{n}=105)\end{array}$ & $\mathrm{p}$ value \\
\hline Age, years & $43.4 \pm 12.7$ & $44.5 \pm 13.1$ & 0.534 \\
\hline Gender, male, n (\%) & $65(61)$ & $63(60)$ & 0.172 \\
\hline BMI, $\mathrm{kg} / \mathrm{m}^{2}$ & $22.5 \pm 2.1$ & $22.8 \pm 2.6$ & 0.098 \\
\hline HTN, n (\%) & $40(38)$ & $38(36)$ & 0.169 \\
\hline $\mathrm{DM}, \mathrm{n}(\%)$ & $20(19)$ & $23(12)$ & 0.158 \\
\hline Hyperlipidemia, n (\%) & $7(6)$ & $9(8)$ & 0.249 \\
\hline Smoking, n (\%) & $47(44)$ & $42(40)$ & 0.365 \\
\hline White blood cell count, $10^{3}(\mu \mathrm{L})$ & $6.4 \pm 1.6$ & $8 \pm 3.2$ & $<0.001$ \\
\hline Neutrophil, $10^{3}(\mu \mathrm{L})$ & $4.1 \pm 2.2$ & $5.5 \pm 2.2$ & $<0.001$ \\
\hline Lymphocyte, $10^{3}(\mu \mathrm{L})$ & $1.1 \pm 0.3$ & $0.9 \pm 0.3$ & $<0.001$ \\
\hline Monocyte, $10^{3}(\mathrm{uL})$ & $0.53 \pm 0.3$ & $0.61 \pm 0.3$ & 0.654 \\
\hline Platelet, $10^{3}(\mu \mathrm{L})$ & $234 \pm 77$ & $244 \pm 80$ & 0.278 \\
\hline Hemoglobin, g/dl & $13 \pm 1.6$ & $12.4 \pm 1.7$ & 0.208 \\
\hline Glucose, mg/dl & $96.0 \pm 8.5$ & $95.5 \pm 9.2$ & 0.426 \\
\hline Aspartate aminotransferase, IU/1 & $29.9 \pm 7.6$ & $27.9 \pm 9.7$ & 0.348 \\
\hline Alanine aminotransferase, IU/l & $29.5 \pm 9.4$ & $28.2 \pm 15.5$ & 0.485 \\
\hline Creatinine $\mathrm{mg} / \mathrm{dl}$ & $0.87 \pm 0.22$ & $0.86 \pm 0.23$ & 0.532 \\
\hline Sodium, mEq/L & $137 \pm 3$ & $137.1 \pm 3.1$ & 0.437 \\
\hline Calcium, mg/dl & $9.40 \pm 0.55$ & $9.45 \pm 0.57$ & 0.619 \\
\hline Potassium, mmol/L & $4.2 \pm 0.3$ & $4.1 \pm 0.3$ & 0.455 \\
\hline Neutrophil to lymphocyte ratio & $3.7 \pm 1.6$ & $6.1 \pm 2.7$ & $<0.001$ \\
\hline $\mathrm{C}$-reactive protein, $\mathrm{mg} / \mathrm{L}$ & $10.6 \pm 6.8$ & N.A & N.A \\
\hline Ferritin, ng/mL & $208 \pm 98$ & N.A & N.A \\
\hline D-dimer, $\mathrm{ng} / \mathrm{mL}$ & $350 \pm 210$ & N.A & N.A \\
\hline
\end{tabular}

$\mathrm{p}$ values $<0.05$ were defined as statistically significant

Data are given as mean $\pm \mathrm{SD}$, median or $\mathrm{n}(\%)$

COVID-19 coronavirus disease 2019, BMI body mass index, DM diabetes mellitus, HTN hypertension, N.A. not applicable
$3.7 \pm 1.6)$ were significantly higher in the COVID-19 group than the control group. Lymphocyte counts $\left(1.1 \pm 0.310^{3}\right.$ $\mu \mathrm{L}$ vs. $\left.0.9 \pm 0.310^{3} \mu \mathrm{L} ; \mathrm{p}<0.001\right)$ were significantly lower in the COVID-19 group than the control group. Mean levels of C-reactive protein (CRP), ferritin and D-dimer were higher than normal values in only patients with COVID19. In addition, there was no difference between the groups in terms of other laboratory findings $(p>0.05)$. Echocardiographic findings evaluated 3 months after the diagnosis of COVID-19 are shown in Table 2. Heart rate, LV wall diamaters, LVEF and LAD were not different between the two groups ( $p>0.05$ ). RV diamaters, sPAP and Tissue doppler RV MPI were significantly higher in the COVID-19 patients compared to control group $(\mathrm{p}<0.05)$. TAPSE and RV FAC were significantly lower in the COVID-19 group compared to control group $(\mathrm{p}<0.05)$. RV-GLS $(-19.6 \pm 5.2$ vs. $-15.1 \pm 3.4, \mathrm{p}<0.001)$ and RV-FWLS $(-19.6 \pm 5.2$ vs. $-17.2 \pm 4.4, p<0.001)$ values were significantly lower in the COVID-19 group than the control group. The relationships

Table 2 Electrocardiographic and echocardiographic characteristics of study population \pm

\begin{tabular}{lccr}
\hline Parameters & $\begin{array}{l}\text { Control } \\
(\mathrm{n}=105)\end{array}$ & $\begin{array}{l}\text { COVID-19 } \\
(\mathrm{n}=105)\end{array}$ & p value \\
\hline Heart rate, bpm & $78.7 \pm 8$ & $77.2 \pm 6$ & 0.475 \\
LVEF, \% & $62.5 \pm 1.4$ & $62.7 \pm 1.2$ & 0.234 \\
LVESd, mm & $31.1 \pm 1.9$ & $32.5 \pm 2.3$ & 0.125 \\
LVEDd, mm & $45.2 \pm 16.3$ & $44.1 \pm 2.3$ & 0.109 \\
LVSWT, mm & $10.2 \pm 0.7$ & $10 \pm 0.6$ & 0.302 \\
PWT, mm & $9.2 \pm 0.5$ & $9 \pm 0.6$ & 0.136 \\
E/e' (lateral) ratio & $9.3 \pm 3.5$ & $9.0 \pm 3.1$ & 0.065 \\
LAD (a-p), mm & $34.8 \pm 2.2$ & $35.2 \pm 2.6$ & 0.127 \\
sPAP, mmHg & $23.4 \pm 4.4$ & $28.1 \pm 5.5$ & $<\mathbf{0 . 0 0 1}$ \\
RV mid-diamater, mm & $25.1 \pm 3.1$ & $26.1 \pm 2$ & $\mathbf{0 . 0 4 2}$ \\
RV basal-diamater, mm & $29.3 \pm 3$ & $30.3 \pm 2.9$ & $\mathbf{0 . 0 2 3}$ \\
RV-FAC, $(\%)$ & $46.4 \pm 5.4$ & $43.4 \pm 5.8$ & $<\mathbf{0 . 0 0 1}$ \\
Tissue doppler RV MPI & $0.38 \pm 0.03$ & $0.48 \pm 0.08$ & $<\mathbf{0 . 0 0 1}$ \\
TAPSE, mm & $24 \pm 4.4$ & $22.4 \pm 2.6$ & $<\mathbf{0 . 0 0 1}$ \\
RV S' cm/s & $13.5 \pm 2.1$ & $12.9 \pm 3.5$ & $\mathbf{0 . 0 1 3}$ \\
RV-GLS, (\%) & $-18.5 \pm 3.2$ & $-15.1 \pm 3.4$ & $<\mathbf{0 . 0 0 1}$ \\
RV-FWLS, (\%) & $-19.6 \pm 5.2$ & $-17.2 \pm 4.4$ & $<\mathbf{0 . 0 0 1}$ \\
\hline
\end{tabular}

$\mathrm{p}$ values $<0.05$ were defined as statistically significant

Data are given as mean \pm SD or $(\%)$

$B p m$ beats per minute, $L V E F$ left ventricular ejection fraction, $L V E D d$ left ventricular end-diastolic diameter, LVESd left ventricular endsystolic diameter, $L V S W T$ left ventricular septal wall thickness, $P W T$ posterior wall thickness, $L V M I$ left ventricular mass index, $L A D(a-p)$ left atrium diamater anterior posterior, $R V$ right ventricle, $R V F A C$ right ventricular fractional area change percentage, $R V M P I$ right ventricular myocardial performance index, TAPSE tricuspid annular plane systolic excursion, $S P A P$ systolic Pulmonary arterial pressure, $R V-F W L S$ right ventricle free-wall longitudinal strain, $R V-G L S \mathrm{RV}$ global longitudinal strain 
of the RV-GLS and RV-FWLS values with NLR, D-dimer, CRP, ferritin were evaluated via Spearman's correlation analyses. The results yielded by Spearman's correlation analysis are presented in Table 3. A statistically significant negative correlation relationship was detected between the RV-GLS and RV-FWLS values and NLR, D-dimer, CRP, ferritin.

\section{Discussion}

In this study, right ventricular functions were assessed with 2D echocardiography at third month after mild severity COVID-19 disease. After 3 months follow-up, TAPSE, RVFAC, RV-GLS and RV-FWLS parameters were significantly lower in patients with mild severity COVID-19 disease than healthy controls, however RV diameters, RV-MPI and sPAP values were significantly higher. Negative correlation was detected during active infection period between RV-GLS, RV-FWLS measurements and levels of inflammatory markers including CRP, ferritin, NLR and D-dimer. These results implies that mild severity COVID-19 disease may lead to decrease in RV-GLS and RV-FWLS parameters that is related to levels of CRP, NLR, ferritin and D-dimer.

The outbreak of COVID-19 (coronavirus disease 2019) caused by SARS-CoV-2 (acute respiratory syndrome coronavirus-2) is a continuing global health crisis. It is well known that COVID-19 disease causes multi organ involvement and the presence of cardiac involvement is related to poor outcomes [12]. Several studies reported that COVID-19 related myocardial damage is associated with worse prognosis $[13,14]$. Standard 2D echocardiography is very useful in COVID-19 patients and it allows to assess disease severity, ventricular functions and hemodynamic state non-invasively. 2D speckle tracking echocardiography (2D-STE), may detect myocardial dysfunction and subclinical myocardial

Table 3 Bivariate correlation analysis between right ventricular 2D-STE parameters and inflammatory markers in the COVID-19 group

\begin{tabular}{lll}
\hline Variables & RV-GLS, $(\%)$ & RV-FWLS, $(\%)$ \\
\hline CRP, mg/L & $r=-0.595 p<0.001$ & $r=-0.608 p<0.001$ \\
D-dimer, ng/mL & $r=-0.551 \mathrm{p}<0.001$ & $r=-0.557 \mathrm{p}<0.001$ \\
Ferritin, ng/mL & $r=-0.310 \mathrm{p}=0.001$ & $\mathrm{r}=-0.334 \mathrm{p}<0.001$ \\
NLR & $\mathrm{r}=-0.428 \mathrm{p}<0.001$ & $\mathrm{r}=-0.440 \mathrm{p}<0.001$ \\
PLR & $\mathrm{r}=-0.307 \mathrm{p}<0.001$ & $\mathrm{r}=-0.319 \mathrm{p}<0.001$ \\
LMR & $\mathrm{r}=0.329 \mathrm{p}<0.001$ & $\mathrm{r}=0.340 \mathrm{p}<0.001$ \\
\hline
\end{tabular}

$2 D$-STE two dimensional speckle-tracking echocardiography, $C R P$ C-reactive protein, $N L R$ neutrophil to lymphocyte ratio, $P L R$ platelet to lymphocyte ratio, $L M R$ lymphocyte to monocyte ratio, $R V$ $F W L S$ right ventricle free-wall longitudinal strain: $R V-G L S$ RV global longitudinal strain deformation earlier and more accurate than standard 2D echocardiography [15]. Therefore we used 2D-STE in addition to standard 2D echocardiographic assessment in order to evaluate long term effects of COVID-19 on right ventricular functions.

Mechanisms underlying COVID-19 related right ventricular damage include increased right ventricular afterload and decreased right ventricular contractility due to several factors such as acute respiratory distress syndrome, pulmonary vascular thrombosis, direct viral myocardial damage, hypoxia, inflammatory response and autoimmune damage [16]. Segmental wall motion abnormality or global hypokinesia at right ventricle might develop in COVID19 and these abnormalities are detected more accurately with 2D-STE. Li et al. reported that despite normal left ventricular ejection fraction values, decreased RV-LS is a strong predictor of mortality in COVID-19 patients [17]. In another study, it is demonstrated that decreased RV-GLS values were detected to be strong and independent predictor of poor outcomes in patients with COVID-19 [18]. Baycan et al. expressed that decreased RV-GLS is related to in-hospital mortality in COVID-19 cases [5]. Günay et al. demonstrated that RV-GLS values decreased during active infection in patients with moderate and severe COVID-19 pneumonia [6]. In our study, we detected that RV-GLS and RV-FWLS parameters were decreased at third months after mild severity COVID-19 disease. These results implies that unfavorable cardiovascular effects of mild severity COVID19 disease such as decreased levels of RV-GLS and RVFWLS may persists long term.

Viral infections, including SARS-CoV-2, can cause a severe inflammatory response. CRP, ferritin levels and NLR are important indicators of systemic inflammation in COVID-19 patients. Yenercag et al. showed that CRP levels were high in patients with COVID-19 [19]. In a study investigating the early stage of COVID-19, CRP levels were found to reflect disease severity and should be used as a key indicator for disease monitoring [20]. Yang et al. showed in their study on COVID-19 patients that high NLR could be considered as an independent biomarker to show poor clinical results [21]. In a meta-analysis by Lagunas-Rangel, they showed that increased NLR levels in COVID-19 patients may indicate poor prognosis [22]. Elevated ferritin levels have been found to significantly correlate with disease severity in COVID-19 infected patients [23]. A prior study found that, compared to moderate cases of COVID-19, severe cases had higher ferritin levels [24]. In our study, mean values of CRP and ferritin levels which were measured at the time of COVID-19 diagnosis established were detected higher than normal cut-off values and NLR value was detected higher in COVID-19 patients than normal population. Additionally, negative correlation between inflammatory markers including CRP, ferritin and NLR levels during active infection and 
long term echocardiographic parameters such as RV-GLS, RV-FWLS detected. These findgins supports that there is a possible relationship between the severity of inflammation during acute infection and long term RV-GLS and RV-FWLS measurements. In addition, these results demonstrates that COVID-19 disease may lead to permanent long term cardiovascular effects even its severity was mild.

Uncontrolled inflammatory response which develops during the COVID-19 disease course causes increased production of thrombin and endothelial dysfunction. In addition, COVID-19 related hypoxemia increases blood viscosity and may lead to predisposition for thrombus formation. Increased inflammatory burden and changes in hemostatic pathways results in high D-dimer levels [25, 26]. Lippi et al. reported that D-dimer levels are increased in near half of patients with COVID-19 disease [27]. Zhaohai et al. expressed in a meta-analysis that; raised D-dimer levels are associated with disease severity and mortality [28]. In our study; high D-dimer levels was detected in $36.6 \%$ of the patients with mild severity COVID-19 disease. Pulmonary vascular thrombosis and pulmonary embolism which may present with increased D-dimer levels may be the underlying cause of right ventricular dysfunction in COVID-19 disease. In this study, we demonstrated that D-dimer levels assessed in the acute stage of the infection was negatively correlated with long term RV-GLS and RV-FWLS measurements. These results implies that high values of $\mathrm{D}$-dimer during the acute infection may be associated with decrease in RV-GLS and RV-FWLS in long term follow up.

SARS-CoV-2, may cause impaired immune system and uncontrolled inflammatory response in some patients. Prolonged healing period due to local and systemic inflammatory responses may cause long term cardiovascular effects [29]. Long term cardiovascular effects of COVID-19 is still unknown. Therefore, it is important that patients with COVID-19 should be followed up for detection and treatment of possible subclinical cardiovascular effects. In this study; long term effects of mild severity COVID-19 disease which constitute the majority of patients, on right ventricular functions were evaluated. Our findings support that COVID19 might cause long term cardiovascular effects.

\section{Conclusion}

To our knowledge, our study is first to evaluate the long term effects of mild severity COVID-19 disease on right ventricular functions. In our study, it was found that RV-GLS and RV-FWLS decreased in the long term (third month) follow-up of patients treated for mild severity COVID-19 disease. Additionally, it is demonstrated that increased CRP, NLR, PLR, ferritin and D-dimer levels during acute infection is related to decreased RV-GLS and RV-FWLS values.
2D- speckle tracking echocardiography may be used for both detection and follow-up for possible long term right ventricular dysfunction after mild severity COVID-19 disease. These findings demonstrate the need for new large-scale, prospective studies evaluating the long term cardiovascular effects of COVID-19 disease.

\section{Limitations}

The data was derived from a single center, and the sample size was relatively small. Other inflammatory markers including interleukin-6, erythrocyte sedimentation rate etc. were not evaluated. Strain echocardiographic evaluation was only performed for right ventricular functions; however strain of left sided chambers were not measured. Due to the absence of echocardiographic datas before COVID-19 disease; it is difficult to determine that myocardial deformation was either present before the COVID-19 disease or developed after the disease. In addition, excluding patients with increased troponin levels restricts the reflection of study results to large populations. Confirmation of RV dysfunction by cardiovascular magnetic resonance imaging could strengthen our results.

\section{Declarations}

Conflict of interest The authors declare that they have no conflict of interest.

\section{References}

1. Guan WJ, Ni ZY, Hu Y et al (2020) Clinical characteristics of coronavirus disease 2019 in China. N Engl J Med 382:1708-1720

2. Kang Y, Chen T, Mui D et al (2020) Cardiovascular manifestations and treatment considerations in COVID-19. Heart (British Cardiac Society) 106:1132-1141

3. Zeng F, Huang Y, Guo Y et al (2020) Association of inflammatory markers with the severity of COVID-19: a meta-analysis. Int J Infect Dis 96:467-474

4. Ozen M, Yilmaz A, Cakmak V et al (2021) D-dimer as a potential biomarker for disease severity in COVID-19. Am J Emerg Med 40:55-59

5. Baycan OF, Barman HA, Atici A et al (2021) Evaluation of biventricular function in patients with COVID-19 using speckle tracking echocardiography. Int J Cardiovasc Imaging 37:135-144

6. Günay N, Demiröz Ö, Kahyaoğlu M et al (2021) The effect of moderate and severe COVID-19 pneumonia on short-term right ventricular functions: a prospective observational single pandemic center analysis. Int J Cardiovasc Imaging. https://doi.org/10.1007/ s10554-021-02171-w

7. Barman HA, Atici A, Tekin E et al (2021) Echocardiographic features of patients with COVID-19 infection: a cross-sectional study. Int J Cardiovasc Imaging 37:825-834

8. World Health Organization (2021) COVID-19 Clinical management: living guidance. https://www.who.int/publications/i/item/ WHO-2019-nCoV-clinical-2021-1. Accessed 25 Jan 2021 
9. Republic of Turkey Ministry of Health (2020) COVID-19 Rehberi [online]. https://covid19.saglik.gov.tr/Eklenti/39061/0/covid19rehberieriskinhastatedavisipdf.pdf. Accessed 12 Oct 2020

10. Lang RM, Badano LP, Mor-Avi V et al (2015) Recommendations for cardiac chamber quantification by echocardiography in adults: an update from the American Society of Echocardiography and the European Association of Cardiovascular Imaging. Eur Heart J Cardiovasc Imaging 16:233-271

11. Voigt JU, Pedrizzetti G, Lysyansky P et al (2015) Definitions for a common standard for 2D speckle tracking echocardiography: consensus document of the EACVI/ASE/industry task force to standardize deformation imaging. J Am Soc Echocardiogr 28:183-193

12. Akbarshakh A, Marban E (2020) COVID-19 and the heart. Circ Res 126:1443-1455

13 Barman HA, Atici A, Sahin I et al (2020) Prognostic significance of cardiac injury in COVID-19 patients with and without coronary artery disease. Coron Artery Dis. https://doi.org/10.1097/MCA. 0000000000000914

14. Frattini S, Maccagni G, Italia L, Metra M, Danzi GB (2020) Coronavirus disease 2019 and cardiovascular implications. J Cardiovasc Med 21:725-732

15. Picard MH, Weiner RB (2020) Echocardiography in the time of COVID-19. J Am Soc Echocardiogr 33:674-675

16. Lan Y, Liu W, Zhou Y (2021) Right ventricular damage in COVID-19: association between myocardial injury and COVID19. Front Cardiovasc Med. https://doi.org/10.3389/fcvm.2021. 606318

17. Li Y, Li H, Zhu S et al (2020) Prognostic value of right ventricular longitudinal strain in patients with COVID-19. JACC Cardiovasc Imaging 13:2287-2299

18. Stockenhuber A, Vrettos A, Androschuck V et al (2021) A pilot study on right ventricular longitudinal strain as a predictor of outcome in COVID-19 patients with evidence of cardiac involvement. Echocardiography 38:222-229

19. Yenercag M, Arslan U, Seker OO et al (2019) Evaluation of $\mathrm{P}$-wave dispersion in patients with newly diagnosed coronavirus disease 2019. J Cardiovasc Med (Hagerstown) 22:197-203
20. Wang L (2020) C-reactive protein levels in the early stage of COVID-19. Med Mal Infect 50:332-334

21. Yang AP, Liu JP, Tao WQ, Li HM (2020) The diagnostic and predictive role of NLR, d-NLR and PLR in COVID-19 patients. Int Immunopharmacol 84:106504

22. Lagunas-Rangel FA (2020) Neutrophil-to-lymphocyte ratio and lymphocyte-to-C-reactive protein ratio in patients with severe coronavirus disease 2019 (COVID-19): a meta-analysis. J Med Virol 92:1733-1734

23. Colafrancesco S, Alessandri C, Conti F, Priori R (2020) COVID19 gone bad: a new character in the spectrum of the hyperferritinemic syndrome? Autoimmun Rev 19:102573

24. Taneri PE, Gómez-Ochoa SA, Llanaj E et al (2020) Anemia and iron metabolism in COVID-19: a systematic review and metaanalysis. Eur J Epidemiol 35:763-773

25. Channappanavar R, Perlman S (2017) Pathogenic human coronavirus infections: causes and consequences of cytokine storm and immunopathology. Semin Immunopathol 39:529-539

26. Selcuk M, Cinar T, Gunay N et al (2021) Comparison of D-dimer level measured on the third day of hospitalization with admission D-dimer level in predicting in-hospital mortality in COVID-19 patients. Medeni Med J 36:1-6

27. Lippi G, Plebani M (2020) Laboratory abnormalities in patients with COVID-2019 infection. Clin Chem Lab Med 58:1131-1134

28. Zheng Z, Peng F, Xu B et al (2020) Risk factors of critical \& mortal COVID-19 cases: a systematic literature review and metaanalysis. J Infect 81:e16-e25

29. Becker RC (2020) Anticipating the long-term cardiovascular effects of COVID-19. J Thromb Thrombolysis 50:512-524

Publisher's Note Springer Nature remains neutral with regard to jurisdictional claims in published maps and institutional affiliations. 